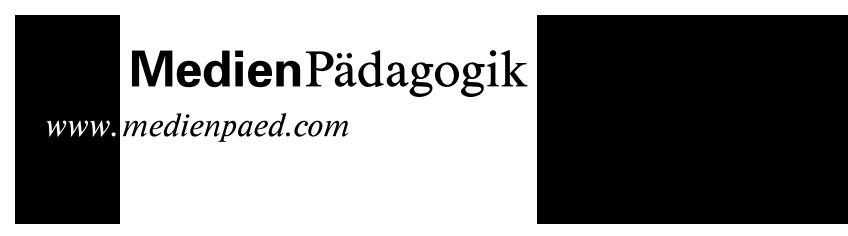

Wolfgang Müskens und Isabel Müskens

24.10.2002

\section{Provokative Elemente einer Didaktik internetgestützter Lehr-Lernarrangements}

Onlinebasiertes kollaboratives Lernen erfordert eine neue Rolle der Lehrenden und damit verbunden auch neue didaktische Methoden. Ausgehend vom Begriff der Provokation wird eine solche Didaktik in Abgrenzung zur Vorstellung eines ausschliesslich selbstgesteuerten Lernens entwickelt. Provokation wird in diesem Zusammenhang als die intendierte Konfrontation eines Lernenden mit einstellungskonträrem Material verstanden. Die Grundlagen der hier vorgestellten Didaktik werden aus einer Vielzahl theoretischer Wurzeln und empirischer Forschungsergebnisse hergeleitet: Es wird auf die Beziehung zum Kompetenzbegriff, zur Systemtheorie, zur Emotionspsychologie, zur Induzierung kognitiver Konflikte, zur Dissonanztheorie und zu Kellys Theorie der persönlichen Konstrukte eingegangen. Als konkrete Methoden der provokativen Didaktik für das E-Learning werden u. a. provokative Statements, das Induzieren einstellungskonträrer Perspektiven und eine an die «Fixed-Role-Therapy» angelehnte Aufgabenstellung diskutiert. Die Methoden der provokativen Didaktik bedeuten eine hochgradige Individualisierung des Lernens und eine Intensivierung des Verhältnisses zwischen Lerner und Lehrendem.

\section{Einleitung}

Lernen und Lehren in internetgestützten Lehr-Lernarrangements stellt hohe Anforderungen an die Akteure. Lernen in Online-Umgebungen erfordert von Lehrenden wie Lernenden den Erwerb neuer Kompetenzen im Umgang mit den verschiedenen medialen Möglichkeiten, aber auch in der Überwindung der bestehenden Beschränkungen. Die mit diesem Kompetenzerwerb einhergehende Entwicklung eines neuen Rollenverständnisses wird in einer ganzen Reihe neuerer Publikationen (u. a. Baltes 2001;
Duggleby 2000; Müskens 2001; Rautenstrauch 2001) eingehend beschrieben. Die Rolle des beinahe omnipotenten Lehrenden hat in virtuellen Lernräumen ausgedient, da sie sich hier weder für Lernende noch für Lehrende in befriedigender Form umsetzen lässt. Auf Seiten der Lernenden ist eine konsumorientierte, unmündige Grundhaltung, wie sie auch in Präsenzseminaren häufig anzutreffen ist, zu überwinden.

Seltener als diese Verschiebungen im sozialen Rollenverständnis wird hingegen thematisiert, welchen Raum internetgestützte Lernarrangements für neue didaktische Konzepte bieten, die deren Möglichkeiten einer individualisierten Kommunikation und zielgerichteten Intervention zwischen Lehrenden und Gruppen von Lernenden sowie einzelnen Lernenden einbeziehen. Einen Anstoss zu einer verstärkten Diskussion solcher didaktischer Möglichkeiten soll das im Folgenden vorgestellte Konzept einer provokativen Didaktik geben.

\section{Provokation als methodisches Element in der Bildung}

Provokation ist als ein methodisches Element des Schulunterrichts in Form von provokativen Fragen und Aufgabenstellungen schon seit den 70er Jahren bekannt und findet auch heute noch in Publikationen zu Unterrichtsmethoden Aufnahme (z. B. Meyer 2000). Die Provokation wird in diesem Rahmen meist als Mittel zur Motivierung eines Lernenden in Richtung auf ein bestimmtes (Lern-)Ziel verstanden. «Der Lehrer als Initiator von Lernakten soll - so könnte man die Grundthese formulieren - den Schüler zum Lernen provozieren» (Kunert/ Zitterbart, 1978). Die Umsetzung der Provokation erfolgt hier in Form von Aufgaben, die beim Lernenden einen hohen Ansporn zu deren Lösung hervorrufen sollen. Mittel hierzu sind u. a. Nähe zu Alltagsproblemen, Einbeziehung der Lernenden und ihrer Ideen sowie Belohnungen bei erfolgreicher Lösung.

Ein sehr viel weitergehendes Verständnis von Provokation im Unterricht findet sich bei Giesecke (1973). Giesecke versteht unter Provokation die Herausforderung von vorhandenen Einstellungen und Verhaltensweisen. Die Provokation soll dem Phänomen entgegen wirken, dass Lernende etwas, das sie als ausserhalb des schulische Rahmens bedeutungslos bewerten trotzdem oberflächlich «lernen», allein weil später Leistungstests hierzu erfolgen. Erklären lässt sich dies nach Giesecke mit einem Widerstand gegen neue Überzeugungen: «Alle Individuen haben die Neigung, an den einmal erworbenen Einstellungen und Urteilen nach Möglichkeit festzuhalten; das gilt vor allem für solche, die vom Individuum aus Gründen 
seiner Selbstdarstellung bzw. der Aufrechterhaltung seiner Identität für besonders wichtig gehalten werden. Informationen nun, die diesen Einstellungen und Urteilen widersprechen, werden entweder abgelehnt oder so uminterpretiert, so dass sie mit den vorliegenden Einstellungen konform werden, oder sie werden bloss verbal nachvollzogen, ohne dass sie die Urteile und Einstellungen verändern oder überhaupt mit ihnen integriert werden.»

Als Formen der Provokation, die dazu führen, dass der Lernende die Distanz zum Gelernten überwindet, nennt Giesecke die Durchführung sozialpsychologischer Tests mit den Lernenden, die gemeinschaftliche gesellschaftliche Provokation von Lehrenden und Lernenden sowie die Provokation der Lernenden durch das Verlangen sinnloser Tätigkeiten ohne Begründung.

Das im Rahmen dieses Artikels zum Ausdruck kommende Verständnis von Provokation ähnelt dem vom Giesecke darin, dass das Ziel der Provokation die Herausforderung bestehender Einstellungen und Urteile sowie die Überwindung der Distanz zwischen Lerninhalten und Lernenden ist. Im Gegensatz zu Giesecke, dessen Ideen geprägt sind durch den Bereich der politischen Bildung und dessen Zielsetzung die Herausforderung gesellschaftlicher Einstellungen war, soll hier die Provokation in das Konzept der Kompetenzentwicklung und das Umfeld der berufsfeldorientierten Erwachsenbildung eingebettet werden. Dies beinhaltet zunächst vor allem eine Ausdehnung der Reichweite des Lehr-Lerngeschehens auf die dem Handeln im Berufsfeld zugrunde liegenden Dispositionen. Hiermit ist automatisch auch der Bereich des Sach- und Methodenwissens einbezogen, im Gegensatz zum erstgenannten Ansatz fokussiert die Provokation hier aber nicht direkt auf eine Erhöhung des Ansporns für dessen Erwerb, sondern auf die Entwicklung von «Competence, defined as the ability to produce successful behaviours in non-standardized situation» (Westera 2001, S. 82). Als Methoden der Provokation, die eine Kompetenzentwicklung beim Lernenden unterstützen können sind Interventionsverfahren geeignet, die dem Lernenden Erfahrungen und Erkenntnisse ausserhalb des bevorzugten, durch seine Dispositionen geprägten, Wahrnehmungshorizontes ermöglichen. Diese werden im Abschnitt «Methoden der provokativen Didaktik» ausführlich beschrieben.

\section{Definition einer provokativen Didaktik}

«Provokative Didaktik ist die intendierte Konfrontation eines Lernenden mit einstellungskonträrem Material»

[intendiert] Ein Akteur des Lehr-Lernarrangements (in der Regel der Lehrende) interveniert aktiv, absichtlich und zielgerichtet in den Lernprozess. Es genügt dieser Definition nicht, wenn das Setting insgesamt so konstruiert ist, dass der Lernende potenziell die Möglichkeit hat, auf einstellungskonträres Material oder Ansichten zu stossen. Über eine solche zufällige Konfrontation geht das intendierte Provozieren von Einstellungskollisionen hinaus.

[einstellungskonträr] Wir nehmen an, dass der Lernende nicht als unbeschriebenes Blatt (tabula rasa) in den Lernprozess kommt, sondern aufgrund seiner bisherigen Erfahrungen und Geschichte Vorwissen, Werte und Einstellungen mitbringt. Eine Kompetenzentwicklung im Sinne von persönlichem Wachstum und Erweiterung des Erfahrungshorizontes ist nur möglich, wenn diese (Vor-)Einstellungen des Lernenden in Frage gestellt werden.

[Konfrontation] Damit der Lernende neue Vorstellungen ausserhalb seines bisherigen Konstruktsystems wahrnimmt, seine bisherigen Einstellungen ggf. modifiziert bzw. korrigiert und schliesslich durch eine Synthese seiner bisherigen mit neuen Einstellungen zur einem erweiterten Verständnis gelangen kann, ist es erforderlich, dass er sich mit dem Konstruktsystem der neuen Vorstellung auseinandersetzt, es elaboriert und erfolgreich mit realen Erfahrungen in Verbindung bringt. Diese Aufgabe ist für den Lernenden anstrengend und oftmals mit negativen Emotionen verbunden. Der Lehrende muss bei der Konfrontation abwägen zwischen Druck auf den Lernenden und dessen Freiwilligkeit, um einerseits dessen ausreichende Beschäftigung mit dem einstellungskonträren Material zu gewährleisten, ohne andererseits dessen Reaktanz herauszufordern.

[Material] Es erscheint uns auch für die Zukunft unrealistisch bzw. unökonomisch, dass Lernen ausschliesslich anhand realer, praktischer Erfahrungen stattfinden wird. Die indirekte Weitergabe von Vorstellungen und Erfahrungen über Lernmaterialien (Texte, multimediale Darstellungen, interaktive Szenarien) kann durch Lernen im Funktionsfeld nicht vollständig ersetzt werden. Das Verstehen der Vorstellungen anderer durch das Nachvollziehen ihrer schriftlich (oder graphisch bzw. multimedial) dargelegten Argumente erscheint uns nach wie vor als ein probates Mittel zur 
Weiterentwicklung des eigenen Verständnisses und damit als ein Schritt zur persönlichen Kompetenzentwicklung.

Die Einstellung, auf der diese Definition von provokativer Didaktik basiert, wird sehr treffend von Mills (1996) in einem Kongressbeitrag beschrieben: «The goals of provocative teaching are grounded in a conceptual framework of critical thinking as well as in an understanding and appreciation of the many psychological processes that influence mental life. Within that context, I believe that the teacher's strategy should be designed to provoke or pique students to think; that is, to analyse the grounds of their beliefs, which can be directly applied to their personal lives. Knowledge without personal meaning is passionless, while personal belief without knowledge is blind. I maintain a fundamental teaching standard - that the passive intellect is unacceptable.»

\section{Einsatz provokativer didaktischer Elemente in internetgestützten Lehr-Lernarrangements}

Internetgestützte Lehr-Lernarrangements, die kommunikations- und kollaborationsorientiert gestaltet sind, stellen eine ideale Grundlage für den Einsatz provokativer Lehrmethoden dar. Dies beruht auf folgenden Merkmalen eines solchen Settings:

- Durch die Verschriftlichung und Speicherung sämtlicher Kommunikationsbeiträge hat der Lehrende die Möglichkeit, sich ein sehr genaues Bild vom einzelnen Lernenden, dessen Einstellungen, Stärken und Defiziten sowie Lernentwicklungen zu machen.

- Durch die Rollenverschiebung des Lehrenden hin zum Lernbegleiter und Initiator von Kollaborations- und Kommunikationsprozessen kann der Lehrende die Lernenden nicht nur in auf sich gerichteten Interaktionen erleben, sondern auch in den Interaktionen mit einzelnen Peers oder der Gruppe.

- Im Gegensatz zum Präsenzunterricht bieten internetgestützte Settings meist eine Fülle von Kommunikationsmitteln an, mit deren Hilfe einzelne Teilnehmer oder Teilnehmergruppen angesprochen werden können. Damit ist eine Basis für sehr individualisierte Lernbegleitung geschaffen. Zudem sind die meisten Kommunikationsmittel (Foren, Mails, Boards) asynchron organisiert, so dass Reaktionen auf Geschehnisse zeitversetzt (und nach gründlicher Überlegung) erfolgen können.

In der Praxis scheinen diese Merkmale internetgestützter Lehr-Lernarrangements, die aus didaktischer Perspektive den eigentlichen Mehrwert dieser Technologien ausmachen, allerdings häufig unterbewertet zu werden. Das Hauptinteresse bei der Verwendung solcher Settings richtet sich meist auf die bequeme, ortsunabhängige Distribution von mehr oder weniger aufwendig gestalteten Lernmaterialien. Die Kommunikationsmittel werden als schlichte Kanäle für Rückfragen zu den Materialien betrachtet und die stattfindenden Lernprozesse auf die reine Vermittlung von Wissen reduziert.

Ganz im Gegensatz hierzu ist eine provokative Didaktik in internetgestützten Lehr-Lernarrangements ohne eine professionelle Nutzung der Kommunikationsmittel und ohne professionelle diagnostische Fähigkeiten der Lehrenden zur Beurteilung von Einstellungen und Lernforschritten undenkbar. Der Einsatz provokativer Methoden ist nur dann erfolgsversprechend, wenn er zielgerichtet und in einem individualisierten Betreuungsverhältnis mit dem einzelnen Lernenden oder einer ausgesuchten Gruppe von Lernenden stattfindet. Ist dies nicht gegeben, ist die Gefahr gross, dass eine Provokation Verwirrung bis Verärgerung unter den Lernenden auslöst oder gänzlich ignoriert wird. Ein Plädoyer für den Einsatz provokativer Methoden in internetgestützten Lehr-Lernumgebungen ist somit zugleich ein Plädoyer für die Realisierung individualisierter Förderstrategien, die Bezug nehmen auf die Entwicklung des jeweiligen Lernenden.

Die hier dargestellte provokative Didaktik ist intendiert als Ergänzung zu anderen vielversprechenden Ansätzen zur Gestaltung internetgestützter Lehr-Lernarrangements wie z. B. Projektlernen, handlungsorientiertes Lernen und soziales Lernen. Die Methoden der provokativen Didaktik können in diese Ansätze sinnvoll integriert werden und diese damit bereichern, sollen sie aber keinesfalls gänzlich ersetzen.

\section{Zur Begründung einer provokativen Didaktik}

Kompetenzentwicklung statt Vermittlung von Sach- und Methodenwissen Sich beschleunigender technologischer Wandel und zunehmende Diversifizierung und Spezialisierung beruflicher Anforderungen stellen die bisherige Gestaltung der beruflichen Weiterbildung in Frage. Die Unvorhersehbarkeit zukünftiger beruflicher Anforderungen verlangt über konkrete Fachkenntnisse und -wissen hinaus die Fähigkeit, sich auf neue, unbekannte Situationen einzustellen, sich selbständig neues Wissen anzueignen, sein eigenes Vorgehen kritisch zu reflektieren und ggf. zu korrigieren und schliesslich, das eigene Handeln selbstständig zu planen und motiviert umzusetzen. In zunehmendem Masse werden also in vielen Berufsfeldern 
Selbstorganisationsfähigkeiten gefordert. Diese Selbstorganisationsdispositionen werden von Erpenbeck und Heyse (1999) als «Kompetenzen» definiert. Kompetenzentwicklung bedeutet somit die Veränderung latenter Dispositionen; zielgerichtete Provokation kann eine Methode sein, solche Veränderungen einzuleiten.

Die wachsende Bedeutung der Kompetenzentwicklung gegenüber klassischer Wissens- und Fertigkeitsvermittlung hat weitreichende Folgen. «Durch die erhöhte Bedeutung sozialer und personaler Kompetenzen für Prozesse des «Leben-lernens' gegenüber wertfrei vermittelbarem Sach- und Methodenwissen tritt die Aneignung und Interiorisation von Werten in den Vordergrund. (...) Dies kann aber nur geschehen, indem ihm (dem Lernenden) ermöglicht wird, sein eigenes Wertesystem zu erkennen, zu analysieren und mit der beratenden Hilfe eines Trainers in einer sozialen Lernsituation einer Überprüfung auf Realitätsangemessenheit zu unterziehen. Erst wenn er selbst erkannt hat, dass seine bisherigen, handlungsleitenden Einstellungen seinen persönlichen Fortschritt hemmen, ist er bereit Bequemlichkeiten und Voreingenommenheiten zu verlassen und sich weiter zu entwickeln» (Erpenbeck/ Heyse 1999, S. 125). Damit gerät die Veränderung von Einstellungen und Werten in den Mittelpunkt des Lerngeschehens.

Keine Veränderung ohne Labilisierung

Erpenbeck und Heyse (1999) betonen den Zusammenhang zwischen biographischen Labilisierungen und Kompetenzentwicklung. Sie stellen fest, «dass 〈algorithmisch〉 nicht lösbare Entscheidungssituationen die Motoren von Wertentstehung und Wertinteriorisation bildeten. Solche Entscheidungssituationen führen stets zu individuellen Labilisierungen, Instabilitäten, Irritationen, Dissonanzen usw., haben also tatsächlich stets etwas krisenhaftes (...) Entwicklung ist stets auf etwas positiv Bewertetes gerichtet, ist wertbezogen. Krisen stellen damit bisherige Ziele und Werte in Frage» (ebd., S. 221). Innerhalb des von Erpenbeck und Heyse vertretenen Forschungsansatzes zur Kompetenzbiographie, ist die Betrachtung von Labilisierungen daher von herausragender Bedeutung, «da dies stets Situationen sind, in denen Werte interiorisiert und Kompetenzen damit fundiert werden» (ebd., S. 221)

Die Vorstellung, dass eine auf Entwicklung hinzielende Veränderung von Werten und Einstellungen nur durch eine vorhergehende systemdestabilisierende Intervention von aussen erfolgen kann, weist auch Parallelen zur
Theorie der Synergetik von Haken (1988) auf. Die Labilisierung ist hier mit einem Phasenübergang gleichzusetzen, die Intervention in das System entspricht dem Kontrollparameter, der die Destabilisierung der alten Struktur auslöst.

Siebert (1999) verwendet in diesem Zusammenhang den von Maturana und Varela (1987) geprägten Begriff der Pertubation: «Pädagogisch heisst dies: Lehrende können die Lernenden anregen, scheinbar Selbstverständliches infrage stellen, einen Perspektivenwechsel vorschlagen. Ein konstruktives Seminar lebt von Überraschungen, auch von Querdenkern und Verfremdungseffekten. Lernintensiv ist die Erkenntnis, dass vieles auch anders betrachtet werden kann, dass es auch andere Sichtweisen gibt. (...) Wir lernen, dass es nicht nur eine richtige Lösung, sondern mehrere mögliche und vorläufige Lösungen gibt. Pertubation heisst: Die Aufmerksamkeit auf Neues, Ungewöhnliches lenken» (S. 38f.).

\section{Lernen setzt Entlernen voraus}

Staudt und Kriegesmann (1999) zitieren in ihrem vielbeachteten Beitrag «Weiterbildung: ein Mythos zerbricht» einen Vortrag von G. Kirsch, in dem dieser fordert: «Eine Lernkultur, in einer sich dynamisch wandelnden Arbeits- und Lebenswelt kann und darf sich demnach nicht nur darauf beschränken, das Erlernen von immer neuen Kenntnissen und Fertigkeiten $\mathrm{zu}$ ermöglichen und zu fördern. Vielmehr muss es auch ihr Anliegen sein, das Vergessen von alten Kenntnissen und Fertigkeiten zu ermöglichen und zu fördern. Auf den ersten Blick mag es scheinen, dass es völlig überflüssig ist, dies eigens hervorzuheben; vergessen würden wir schon ganz von selbst, dazu bedürfe es keiner Anstrengung; im Zweifel würden wir gar mehr von dem vergessen, was wir kennen und können, als wünschenswert ist. Nun ist gewiss richtig, dass Kenntnisse und Fertigkeiten, die noch von Nutzen sein können, vergessen werden. Allerdings ist auch richtig, dass völlig veraltete Kenntnisse und Fertigkeiten als Ballast mitgeschleppt werden. Und dies hat dann nicht nur zur Folge, dass die Speicherung des (überflüssigen) Alten die Aufnahme von (nützlichem) Neuen behindert oder gar verhindert. Vielmehr muss man auch befürchten, dass die alten, die veralteten Kenntnisse und Fertigkeiten - nur weil sie noch präsent sind - angewendet werden. Es scheint demnach keineswegs überflüssig zu sein, dass eine Lernkultur für eine sich in konstantem Wandel befindliche Arbeits- und Lebenswelt nicht nur die Fähigkeit und Bereitschaft, Neues zu erlernen vermitteln muss; sie muss auch die Bereitschaft und Fähigkeiten 
fördern, Altes aufzugeben, fallen zu lassen, zu vergessen» (S. 38).

Selbst wenn man sich dem mechanistischen Speichermodell des Gedächtnisses, wie Kirsch es offenbar vertritt, nicht anschliessen mag, besitzen seine Forderungen doch zweifellos Gültigkeit, wenn es um Kompetenzentwicklung und damit um die Veränderung von Einstellungen und Werten geht. Weltanschauungen, Wertvorstellungen und Stereotype können den Blickwinkel auf abweichende Meinungen und Vorstellungen verengen und die offene Rezeption neuer Ideen verhindern. Hier muss oft zunächst ein kritisches Infragestellen oder sogar vollständiges Aufgeben überkommener Ansichten stattfinden, bevor neue Einstellungen und Werte aufgebaut werden können.

Dass das Aufgeben bisheriger Überzeugungen und Werte keineswegs nur ein passiver Vergessensprozess ist, wird auch von Staudt und Kriegesmann (1999) erkannt: «Um ein Entlernen zu bewirken, muss sich das Individuum bewusst entscheiden, das bisher Gelernte nicht mehr anzuwenden. Dieses Entlernen entspricht jedoch keinem simplen Ablöseprozess sondern ist ein aufwendiger und für den Betroffenen schwieriger Prozess» (S. 39). Abermals zitieren Staudt und Kriegesmann hierzu Kirsch: «Der Verlust verfestigter Denkrahmen, alter Gewohnheiten und internalisierter Überzeugungen wird von den Mitarbeitern deshalb regelmässig als äusserst schmerzhafter Prozess empfunden» (S. 39).

Es ist bemerkenswert, dass Kirsch hier die negativen Begleitemotionen einer Einstellung- bzw. Wertveränderung klar erkennt. Nichtsdestotrotz fordert er parallel zu einer neuen Lernkultur des Lernens auch eine «Kultur des Verlernens» (S. 39).

\section{Konflikte fördern Verstehen}

Verbreitete Fehlannahmen (Common Misconceptions) sind in einer Vielzahl eindrucksvoller und anschaulicher Studien insbesondere anhand von Schülerstichproben untersucht worden (z. B. von Vosniadou 1994; Baxter 1995; Roth/ Anderson 1988; Anderson/ Smith 1987; Duit 1995). So untersuchten beispielsweise Anderson und Smith (1987) das Verständnis von Fünftklässlern zur Nahrungsaufnahme bzw. -herstellung (Photosynthese) von Pflanzen. Eine verbreitete Fehlannahme der Schüler bestand darin, anzunehmen, dass Pflanzen ebenso wie Tiere ihre Nahrung ausschliesslich aus der Umwelt (z. B. durch die Wurzeln) aufnähmen. Tatsächlich sind Pflanzen in der Lage, durch die Photosynthese ihre Nahrung selbst herzustellen. Roth und Anderson bemühten sich durch entsprechenden
Unterricht dieses Missverständnis der Schüler im Rahmen des Unterrichts auszuräumen. Eine Überprüfung nach mehreren Wochen Unterricht ergab jedoch, dass nur 7\% der Schüler ihre frühere Vorstellung revidiert hatten. Die überwältigende Mehrzahl der Schüler hielt an der bisherigen Vorstellung fest, obwohl sie auf Nachfrage angegeben hatten, den Sachverhalt verstanden zu haben. Anderson und Roth (1989) kommen zu dem ernüchternden Schluss, dass Schüler immer zustimmen, wenn sie gefragt werden, ob sie verstanden haben, ganz unabhängig davon, ob sich ihr Verständnis tatsächlich geändert hat.

Common Misconceptions sind jedoch keineswegs auf Kinder und Jugendliche beschränkt. Auch bei Studierenden des Diplomstudienganges Psychologie fanden wird weitverbreitete Fehlannahmen, z. B. im Bereich der sozialwissenschaftlichen Methodik:

- «Varianzanalysen vergleichen die Varianzen von Variablen.»

- «Mit «Grounded Theory〉 wird eine Theorie überprüft.»

- «Das Beibehalten einer Nullhypothese <beweist> diese.»

- «Der Forscher möchte nicht, dass die Nullhypothese verworfen wird, weil ansonsten seine Theorie widerlegt wird.»

- «Ein signifikantes Ergebnis muss auch bedeutsam sein.»

- «Die Annahme der statistischen Alternativhypothese beweist die inhaltliche Hypothese.»

- «Eine negative Korrelation ist immer ein schwacher Zusammenhang.»

- Common Misconceptions besitzen eine ganze Reihe von problematischen Eigenschaften: Sie

- können über lange Zeit verborgen bleiben,

- sind oftmals schwer zu identifizieren, da Aussagen, die ihnen widersprechen, vom Schüler «umgedeutet» werden und dann mit neuem Gehalt selbst verwendet werden,

- können bei einer strikt linearen Unterrichtsplanung nicht wieder korrigiert werden,

- entstehen bevorzugt bei Frontalunterricht und dem Lernen aus Sekundärliteratur (Zusammenfassungen, Skripte),

- entstehen, wenn Vorstellungen nicht an realen Erfahrungen überprüft werden können.

Als Möglichkeiten zur Auflösung von Fehlannahmen werden u. a. Gegenargumente (Balacheff 1991) und die Konfliktinduzierung (Bell 1983; Underhill 1991) vorgeschlagen.

Die Methode der Konfliktinduzierung fand z. B. in mehreren experimen- 
MedienPädagogik

tellen Settings von Johnston und Scott (1991) Verwendung. Im Mittelpunkt eines dieser Settings stand die verbreitete Fehlannahme unter Schülern, dass die Masse eines festen Stoffes bei einer Auflösung in einer Flüssigkeit verloren gehen, bzw. reduziert würde. Zur Widerlegung dieser Fehlannahme hatten die Schüler die Aufgabe, ein Gefäss mit Wasser während der Auflösung von Zucker zu wiegen.

Die verschiedenen Reaktionsmöglichkeiten auf die Widerlegung einer solchen Fehlannahme, sollen hier anhand des von Johnston und Scott (1991) entwickelten Experimentalparadigmas kurz dargestellt werden:

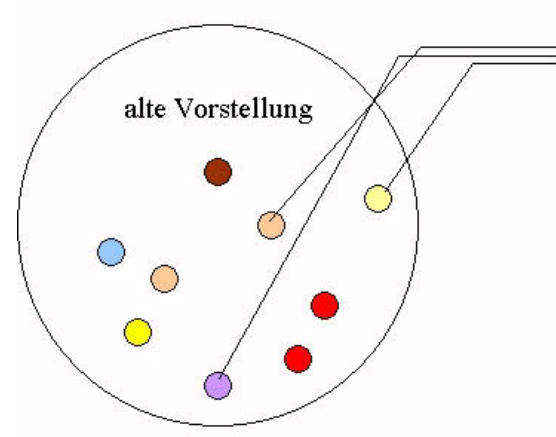

Erfahrungen

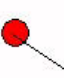

Abb. 1.: Auftreten eines Konfliktes

○ Konflikt

Der Schüler nimmt an, dass feste Stoffe, die in einer Flüssigkeit aufgelöst werden, ihre Masse verlieren (alte Vorstellung). Der Konflikt entsteht, indem das Gefäss während des Auflösens von Zucker gewogen wird (Abb.1).

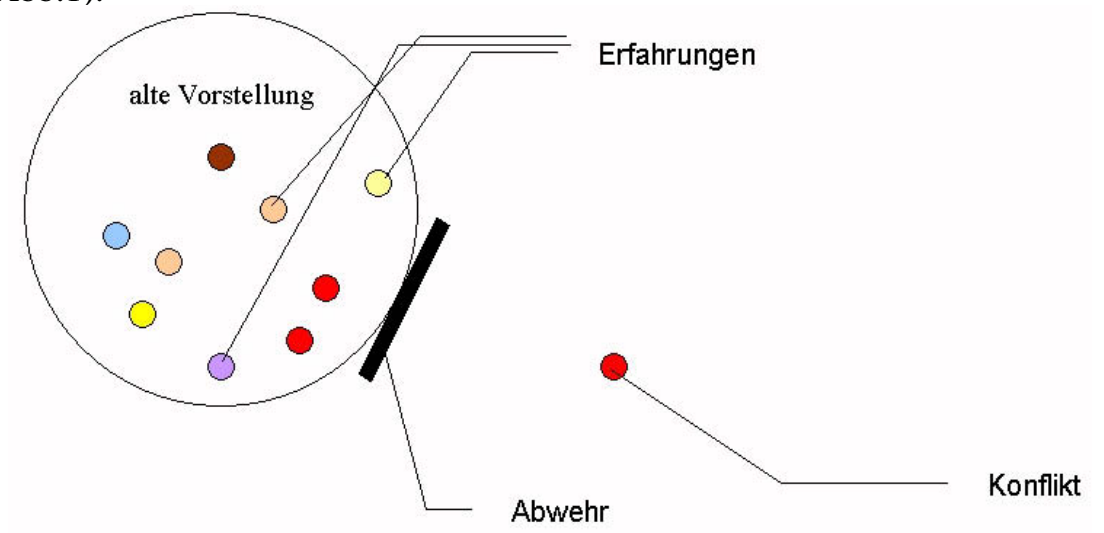

\section{○ Leugnen}

«Die Waage muss defekt sein.»

Die Schüler leugnen die Evidenz, indem sie z. B. das Funktionieren des Messinstrumentes anzweifeln (Abb. 2).

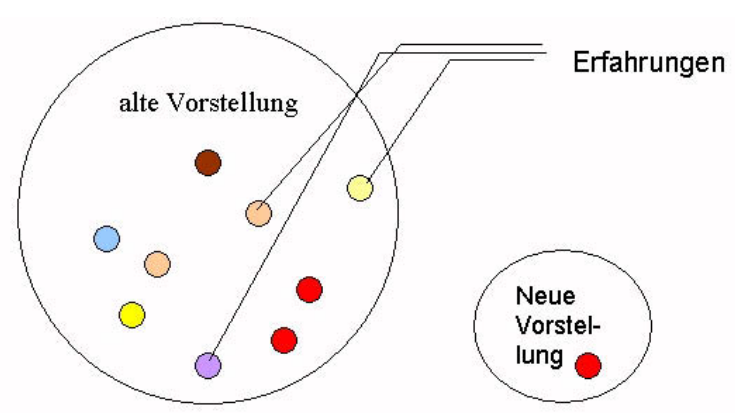

Abb. 3.: Mögliche Reaktion: Entstehung einer begrenzten, neuen Vorstellung

○ Entstehung einer neuen Vorstellung

«Zucker verliert seine Masse nicht, wenn er in einer Flüssigkeit aufgelöst wird. Andere Stoffe können aber trotzdem ihre Masse verlieren (z. B. Wasser, wenn es verdampft.)». Es entsteht eine begrenzte, neue Vorstellung, ohne dass die alte Annahme insgesamt bedroht wird (Abb. 3).

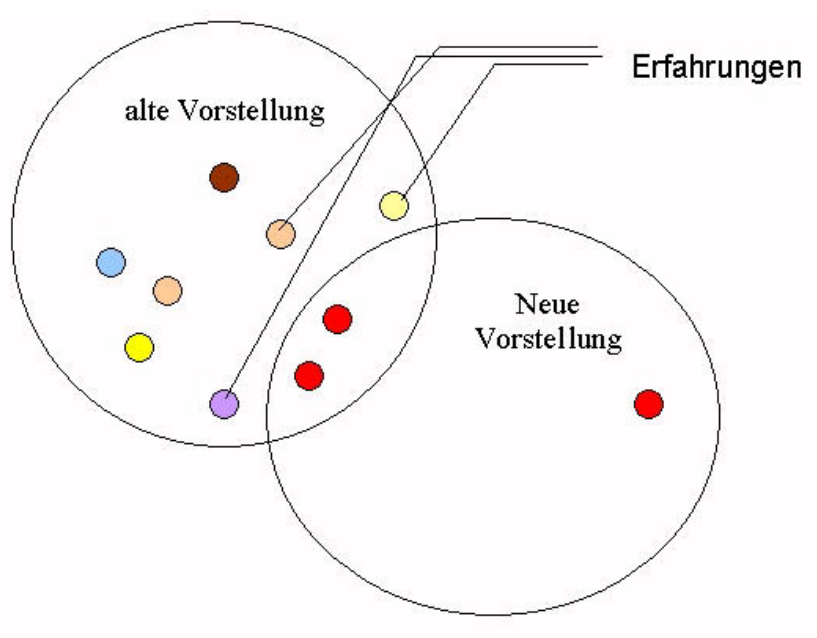

Abb. 4.: Mögliche Reaktion: Dissoziation der Erklärungsmodelle 


\section{○ Dissoziation}

«Die Erklärungen und Experimente aus dem Physikunterricht haben nichts mit der wirklichen Welt zu tun. In Wirklichkeit verlieren aufgelöste Stoffe ihre Masse. In der experimentellen Umgebung der Schule gelten irgendwie künstliche andere Regeln.»

Der Schüler dissoziiert gedanklich die Erfahrungswelten. Es wird eine diskriminierende Bedingung konstatiert, die es ermöglicht, die bisherige Vorstellung zumindest unter entsprechenden Bedingungen aufrecht $\mathrm{zu}$ erhalten (Abb. 4).

○ Revision der alten Vorstellung

«Wenn Zucker seine Masse beim Auflösen in Wasser nicht verliert, verliert möglicherweise auch Wasser seine Masse nicht beim Verdampfen.»

Damit das gedankliche Experimentieren des Schülers dann tatsächlich zu einer neuen, erweiterten, bzw. modifizierten Vorstellung führt (Abb. 5.), müssen i. d. R. allerdings mehrere Prozesse stattfinden:

- Der Schüler muss versuchen, bereits vorhandene Erfahrungen mit der neuen Vorstellung zu erklären. Es muss entschieden werden, ob diese Erfahrungen fortan mit der alten oder mit der neuen Vorstellung erklärt werden sollen, oder ob eine Erklärung mit beiden Vorstellungen möglich ist, die zu übereinstimmenden Vorhersagen führt.

- Der Geltungsbereich der alten Vorstellung muss dann eingeschränkt werden, wenn ihre Vorhersagen nicht mit der neuen Vorstellung übereinstimmen.

- Neue Erfahrungen (z. B. weitere Experimente) müssen stattfinden, die die neue Vorstellung bekräftigen.

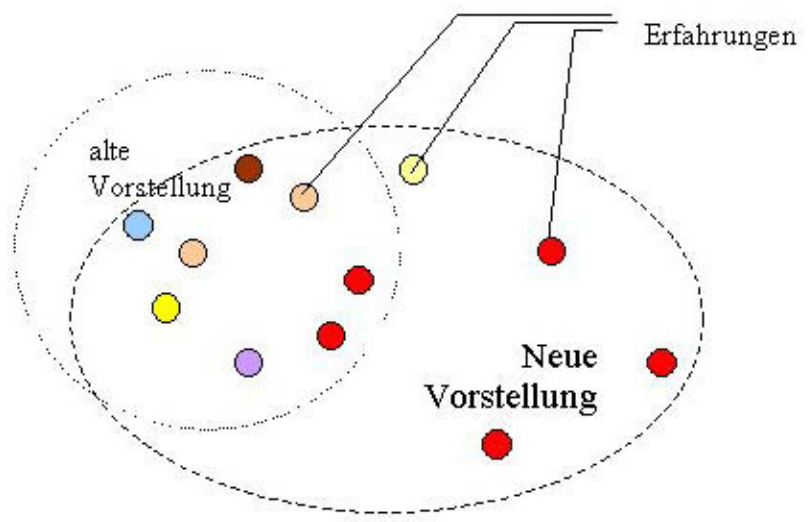

Abb. 5: Mögliche Reaktion: Stabilisierung der neuen Vorstellung
Die gezielte Induzierung von Konflikten wie im Experiment von Johnston und Scott (1991) wird z. B. von Draschoff (2000) als intendierte didaktische Methode vorgeschlagen. Diese konfliktinduzierende Methode ist jedoch mit einer Reihe von Problemen verbunden:

- Die Konfliktinduktion ist nur ein erster Schritt zur Auflösung ungeeigneter Konstrukte und zur Bildung alternativer Vorstellungen. In vielen Fällen wird die neue Erfahrung eher abgeblockt werden oder eine sehr problematische «Dissoziation der Erfahrungswelten» eintreten.

- Die neue Vorstellung kann u. U. in Bezug auf andere Erfahrungen sehr viel unangemessener sein als die alte Vorstellung. Es gibt keine Garantie dafür, dass die neue Vorstellung stärker mit der Vorstellung des Lehrenden übereinstimmt als die alte Vorstellung. («Der Zucker ist irgendwie im Wasser versteckt und hat sich gar nicht gelöst.»)

- Wird die Konflikterfahrung nicht durch weitere Erfahrungen gestützt, wird die neue Vorstellung schon bald vergessen bzw. aufgegeben.

- Wird die Konflikterfahrung nicht auf bereits erlebte Erfahrungen angewendet, so bleibt die neue Vorstellung auf einen sehr engen Erfahrungsbereich beschränkt.

- Die Antizipation aller denkbaren Fehlkonzeptionen durch den Lehrenden bzw. Entwickler einer Lernsoftware ist extrem aufwendig. Für jede denkbare Fehlkonzeption muss eine passende Konflikterfahrung konstruiert werden.

- Die Konflikte werden als Stress erlebt. Schüler mit geringem Stressbewältigungspotential sind nicht in der Lage, die Konflikte produktiv zu nutzen.

Zusammenfassend lässt sich feststellen, dass kognitive Konflikte möglicherweise eine notwendige aber sicherlich keine hinreichende Bedingung für die Veränderung von Anschauungen und Vorstellungen sind.

\section{Lernprozesse und Emotionen}

Anders als die Aneignung von Fachwissen, kann die Veränderung von Einstellungen in hohem Masse von Emotionen begleitet sein. Einstellungen und Emotionen sind ursächlich miteinander verknüpft. Einstellungen werden entweder als affektive Bewertung zu einem Objekt bzw. Thema konzipiert (z. B. Fishbein 1967) oder aber als ein mehrdimensionales Konstrukt, das zumindest eine affektiv-emotionale Komponente beinhaltet (Rosenberg/ Hovland 1960). Eine bekannte eindimensionale Einstellungsdefinition von Petty und Cacioppo (1981) beipielsweise lautet: «Ein- 
stellung ist ein generelles, allgemeines und länger anhaltendes positives oder negatives Gefühl gegenüber einer Person, Objekt oder Thema.»

Wenn im Rahmen der Kompetenzentwicklung nun Einstellungen und deren Änderungen in den Mittelpunkt didaktischer Bemühungen rücken und die Vermittlung wert- und einstellungsneutralen Fachwissens an Bedeutung verliert, so bedeutet dies, dass Lerninhalte und -prozesse viel stärker als bisher emotional besetzt sind.

Eine solche «Emotionalisierung» des Lernens äussert sich z. B. im leidenschaftlichen Vertreten einer Ansicht, in der aggressiven Abwehr einstellungskonträrer Aussagen, in der furchtsamen Annäherung an eine neue Theorie, in der Angst, bislang aus einer falschen Vorstellung heraus gehandelt zu haben, in der Scham des Erkennens eigener Misconceptions aber auch in der Euphorie über ein erweitertes Verstehen oder einen aufregenden neuen Gedanken. Solche Emotionen stellen den Lehrenden vor gänzlich neue Aufgaben und Herausforderungen. (Nicht zuletzt vor die Notwendigkeit, hierbei seine eigenen Emotionen wahrzunehmen und zu kontrollieren.)

Eine emotionale Beteiligung des Lernenden im Lernprozess ist jedoch auch mit positiven Effekten verbunden: Eine Vielzahl von Experimenten belegt, dass emotional getönte Erlebnisse besser erinnert werden als neutrale. So liess schon Peters (1911) Versuchspersonen Alltagsereignisse aufschreiben, die ihnen auf ein Reizwort hin ins Gedächtnis kamen. Anschliessend sollten die Probanden, diese Ereignisse hinsichtlich ihrer affektiven Qualität einstufen. Hierbei wurden 80\% der Ereignisse von den Probanden als gefühlsbetont eingestuft. In einer ähnlichen Untersuchung von Turner und Barlow (1951) wurden die Versuchspersonen 14 Tage nach dieser Erhebung unangekündigt erneut gebeten, sich an die geschilderten Ereignisse zu erinnern. Es zeigt sich, dass die Ereignisse umso besser erinnert wurden, je intensiver deren emotionale Qualität zuvor eingestuft worden war. Es spielte dabei keine Rolle ob es sich um positiv oder negativ emotional besetzte Ereignisse handelte. Und auch Jarvilehto (2000) kommt in seinen Ausführungen zur «theory of the organism-environment system» zu folgender Überzeugung: «Although development goes over positive results, also negative emotions may be a source of development, because they are expressions of real problems in the organization of the organism-environment system. The forced repression of the negative emotions means that the situation is not solved; the disintegrative factors stay in the system and their real causes are veiled. Emotion is the way to have knowledge about the presence of such disturbing factors, and it is of the utmost importance to use this 〈primitive> knowledge.»

Diese Ergebnisse belegen die verbreitete Alltagserfahrung, dass emotional geprägte Ereignisse bzw. Lerninhalte besser behalten und erinnert werden als affektiv neutrales Material.

Selbstgesteuertes Lernen kann zu Schmalspurdenken führen

Selbstorganisierte bzw. selbstgesteuerte Lernformen werden häufig als ein Leitbild der gegenwärtigen Didaktik dargestellt. So beschreiben z. B. Arnold et al. (1998) bezogen auf die berufliche Bildung Selbstregulierung und Selbststeuerung als einen von drei übergreifenden «didaktischen Trends» (S. 22). Selbststeuerung wird hier beschrieben mit «was, wann und wie gelernt wird, bestimmt der Lernende selbst» (S.22).

Dohmen et al. (2001) unterscheiden zwischen selbstorganisiertem und selbstgesteuertem Lernen: «〈Selbstorganisiert» ist das Lernen, wenn es in eigener Zuständigkeit in Gang gesetzt und gestaltet wird, und zwar im Blick auf relevante Aspekte. (...) 〈Selbstgesteuert〉 ist das Lernen, wenn es in einem gegebenen Rahmen oder auf einer vorhandenen Grundlage einzelne, bereits konstituierte Aspekte ausgestaltet» (S. 169). Sie halten das selbstgesteuerte Lernen gar für eine Lösung für «epochale Herausforderungen»: «In einer neuen offeneren Weltsituation und einem erweiterten globalisierten Lebens-, Arbeits- und Medienhorizont mit unerwarteten Anforderungen und vielfältigen Optionen müssen die Menschen ihr Leben und Lernen stärker selbst steuern» (S. 168).

Selbstgesteuertes Lernen gibt dem Lernenden die Möglichkeit, seine Lerninhalte selbst zu bestimmen oder zumindest aus einem Angebot auszuwählen. Dies kann durch eine Modularisierung von Lehrgängen geschehen: «Die Aufgliederung langer planmässiger fachsystematischer Lehrgänge in stärker themen- und problembezogene Module mit alternativen Auswahl und Kombinationsmöglichkeiten ermöglicht es den verschiedenen Lernerinnen und Lernern, sich selbst die Informationen und Lernhilfen herauszusuchen und zusammenzustellen, die sie jeweils nach ihren persönlichen Lernbedürfnissen und situativen Voraussetzungen brauchen» (Dohmen et al. 2001, S. 163f.).

Selbstgesteuertes Lernen verlangt auch eine neue Rolle des Lehrenden. Der Lehrende soll nicht mehr Lehrinhalte vermitteln, sondern zum «Lernermöglicher» werden (Siebert, 1996). Heuer (2001) verdeutlicht das neue Leitbild des Lehrenden: «Der Lernermöglicher soll eher unauffälliger Be- 
rater der Lernenden sein. (...) Auslegungen orientieren sich am Berufsbild des Animateurs (vom Belehren zum Animieren) und des Beraters (vom Fachmann zum Ratgeber). (...) Neu ist, dass es im Lernarrangement kein Machtgefälle mehr geben soll. Der Zugriff auf Wissen und Können wird in die Hand der Lernenden gelegt, die Lehrenden beraten auf Nachfrage» (S. 15f.). Es stellt sich die Frage, ob selbstgesteuertes Lernen, wie es hier beschrieben wurde, zur Kompetenzentwicklung, und damit der Veränderung von Einstellungen und Werten, beitragen kann. Selbstgesteuertes Lernen gibt dem Lernenden (u. a. durch die Auswahl entsprechender Lernmodule) die Möglichkeit, aktiv zu entscheiden, ob er sich mit einstellungskonformen oder -konträrem Material auseinandersetzen möchte.

Ein Anhänger einer Theorie oder eines Paradigmas X (z. B. des Behaviorismus), das teilweise oder vollständig im Widerspruch zu einer Theorie $Y$ (z. B. der Psychoanalyse) steht, kann frei entscheiden, ob er z. B. bei der Lösung eines realen oder realitätsnahen Problems wie gewohnt auf sein bevorzugtes Paradigma zurückgreifen möchte oder aber erstmals eine bislang abgelehnte Theorie zu Rate ziehen möchte.

Ein weiteres Beispiel: Ein Studierender hat bislang in einer Reihe von Prüfungen zu unterschiedlichen Fächern stets einen Schwerpunkt auf die Theorie X gelegt. In einer weiteren Prüfung hat er die Wahl, ob er als Prüfungsschwerpunkt abermals die Theorie $X$ oder aber eine partiell widersprechende Theorie Y wählen möchte.

Die Gefahr selbstgesteuerten Lernens besteht nun darin, dass Anhänger eines Paradigmas, einer Theorie oder gar eines bestimmten Stereotyps, neue Informationen, widersprechende Argumente oder konkurrierende Vorstellungen vermeiden. Eine solche aktive Vermeidung jeglicher Horizonterweiterung könnten wir als Schmalspurdenken beschreiben.

Zur Frage der selektiven Informationssuche bzw. Informationsvermeidung wurden unzählige Untersuchungen durchgeführt. Ein Beispiel hierfür is das Experiment von Mills et al. (1959), bei dem die Versuchspersonen (Studierende) sich zunächst entscheiden sollten, ob sie eine Prüfung lieber in Form einer Klausur oder eines Multiple-Choice-Tests absolvieren wollten. Anschliessend wurden ihnen Artikel vorgelegt, die positive Informationen entweder über die eine oder über die andere Prüfungsform beinhalteten. Die Versuchspersonen sollten einen der Artikel auswählen und lesen. Erwartungsgemäss bevorzugten Studierende, die sich für die Klausur entschieden hatten, Artikel, die sich mit den Vorteilen von Klausuren beschäftigten. Ebenso wurde von Studierenden, die sich für den Multiple-
Choice-Test entschieden hatten, Artikel bevorzugt, die die Vorteile dieser Prüfungsform darstellten.

Die Ergebnisse aus Experimenten zur selektiven Aufmerksamkeit sind nicht immer so eindeutig wie in diesem Fall. Man kann aber zusammenfassend feststellen (vgl. Herkner 1988), dass bei der Auswahl von Informationen immer dann einstellungskonsonantes Material bevorzugt wird, wenn nicht mit der Auswahl einstellungskonträren Materials ein klarer Vorteil verbunden ist. Einstellungsdissonante Informationen werden z. B. dann gewählt, wenn man potentielle Argumente von Diskussionsgegnern kennen lernen möchte.

Selbstgesteuertes Lernen birgt somit die Gefahr selektiver Informationsauswahl. Diese Gefahr potenziert sich, wenn die Auswahl einstellungskonsonanten Materials darüber hinaus mit objektiven (z. B. ökonomischen) Vorteilen verbunden ist. Dies wäre z. B. der Fall, wenn der Lernende durch die Wahl eines bekannten, einstellungskonsonanten Themas eine geringere Schwierigkeit bei einer Hausarbeit oder Klausur zu erwarten hätte.

Die dargestellte Problematik ist keineswegs artifiziell konstruiert: Jeder klinische Psychologe weiss, dass kaum ein Verhaltenstherapeut jemals ein Buch über Psychoanalyse gelesen hat, ebenso wenig wie ein Psychoanalytiker sich mit behavioristischen Vorstellungen auseinandersetzen würde. Den Anhängern beider Paradigmen stünde prinzipiell einer Erweiterung ihres bisherigen Konstruktsystems offen, genutzt wird diese aber nur selten. Eine ehemalige Studentin der Pädagogik berichtete uns, sie habe sich bereits vor ihrem Studium mit der Systemtheorie beschäftigt. Im Studium habe sie nach Möglichkeit nur Seminare und Vorlesungen gewählt, die ebenfalls systemisch ausgerichtet seien. Auch ihre Diplomarbeit habe sie schliesslich sie konsequenter Weise zu einer Anwendung der Systemtheorie geschrieben.

Wenn selbstgesteuertes Lernen dazu führt, dass Lernende nicht mehr mit einstellungskonträrem Material konfrontiert werden bzw. diesem aktiv ausweichen können, besteht die Gefahr einer Fixierung auf bestimmte theoretische Vorstellungen und Paradigmen.

\section{Die Bildung neuer Konstrukte}

Im Mittelpunkt der Theorie der persönlichen Konstrukte von Kelly (1955) steht die Vorstellung, dass Menschen in der Auseinandersetzung mit ihrer Umwelt in ähnlicher Weise Konstruktsysteme aufbauen wie dies auch von Wissenschaftlern bei der Entwicklung und Überprüfung von Theorien ge- 
schieht. Ebenso wie es das Ziel von Wissenschaften ist, Phänomene des Gegenstandsbereiches zu erklären bzw. vorherzusagen, beschreibt Kelly in seinem «Organization Corollary» die Antizipation von Ereignissen als die zentrale Aufgabe eines Konstruktsystems: «Each person characteristically evolves, for his convenience in anticipating events, a construction system ...» (S. 39).

Die Veränderung von Konstruktsystemen wird von Kelly als Lernen charakterisiert. Diese Definition bezieht explizit Prozesse der Kompetenzentwicklung, die mit Einstellungs- und Wertveränderungen verbunden sind, mit ein. Die Veränderung von Konstrukten bzw. Konstruktsystemen geschieht nach Kelly aber keineswegs spontan, sondern ergibt sich durch neue Erfahrungen, die mit dem bisherigen Konstruktsystem nicht vereinbar sind: «it follows that the successive revelation events invites the person to place new constructions upon them whenever something unexpected happens. Otherwise one's anticipations would become less and less realistic. The succession of events in the course of time continually subjects a person's construction system to a validation process. (...) As one's anticipations or hypotheses are successively revised in the light of the unfolding sequence of events, the construction system undergoes a progressive evolution. The person reconstrues. This is experience» (S. 50f.).

Die Veränderung von Konstruktsystemen ist mit negativ besetzten Emotionen verbunden. Kelly (1955) beschreibt spezifische emotionale Zustände als geradezu identisch mit bestimmten Phasen der Konstruktveränderung und definiert daher eine Reihe von Einzelemotionen aus der Theorie der persönlichen Konstrukte heraus:

- Bedrohung «Threat is the awareness of imminent comprehensive change in one's core structures» (S. 361).

- Angst «Anxiety is the recognition that the events with which one is confronted lie outside the range of convenience of one's construct system» (S. 365).

- Feindseligkeit «Hostility is the continued effort to extort validational evidence in favour of a type of social prediction which has already proved itself a failure» (S. 375).

Die grosse Bedeutung von Emotionen bei der Veränderung von Konstruktsystemen, bietet eine Erklärung für den Widerstand, der einem solchen Prozess entgegengesetzt werden kann, bzw. für die Schwierigkeiten, auf die ein Tutor oder Therapeut stossen wird, wenn er versucht, Veränderungen im Konstruktsystems eines Lernenden bzw. Klienten anzuregen.
Für den Aufbau neuer Konstrukte nennt Kelly daher eine Reihe von Bedingungen, die geeignet sind, deren Entstehung zu unterstützen:

1. «Use of fresh elements»

2. «Experimentation»

3. «Availability of validating data»

- zu 1.: Use of fresh elements»

Die Entstehung neuer Konstrukte ist ein empfindlicher Prozess. Bevor es zu einem Konflikt neuer Konstrukte mit bereits bestehenden Konstrukten kommt, sollten die neuen Konstrukte zumindest teilweise stabilisiert, d. h. durch erfolgreiche Anwendung (bei der Vorhersage von Ereignissen) gestützt worden sein. Damit ein neues Konstruktsystem nicht das bestehende bedroht, sollte es anhand solcher Elemente eingeführt werden, die relativ wenig Überschneidung bzw. Konfliktpotential zu bereits bestehenden Konstrukten aufweisen. Im weiteren Verlauf des Validierungsprozesses des neuen Konstruktsystems können dann auch solche Elemente aufgegriffen werden, die inkompatibel zu anderen Konstrukten des Klienten oder Lernenden sind.

- zu 2.: «Experimentation»

Der Klient bzw. Lernende muss die Gelegenheit erhalten, die neuen Vorstellungen oder Verhalten in der Praxis zu erproben. Um ein neues Konstruktsystem zu entwickeln, muss er in die Rolle des Wissenschaftlers rücken, der das neue Konstrukt zunächst als Hypothese aufstellt und deren Implikationen in der Empirie (z. B. in einem Experiment) erforscht. Gelingt die Bestätigung der Hypothese, so wird dies als Unterstützung der Theorie (i. e. des Konstruktsystems) angesehen. Das Experimentieren des Lernenden besteht in der Regel aus einer Anwendung neuer Vorstellungen im praktischen Handeln.

Während Kelly hierbei vorwiegend eine psychotherapeutisch-klinische Anwendung im Sinne des Ausprobierens neuer Verhaltensweisen bzw. Rollen im Alltag betrachtet, steht im Rahmen der provokativen Didaktik ein Experimentieren mit neuen Vorstellungen im Vordergrund. («Was würde sich ergeben, wenn ich die Theorie $\mathrm{X}$, die ich bislang stets abgelehnt habe, auf dieses oder jenes Problem anwenden würde?») 
- zu 3.: «Availability of validating data»

Das Experimentieren mit neuen Vorstellungen bzw. Verhaltensweisen alleine genügt nach Kelly nicht, um die Übernahme neuer Konstrukte zu gewährleisten. Hierzu muss der Lernende zusätzlich die Erfahrung machen, dass die neuen Elemente erfolgreich darin geeignet sind, zukünftige Ereignisse zu antizipieren. Es muss also zu einer Rückmeldung über den Erfolg (oder Misserfolg) des neuen Handelns kommen. Zur Aufgabe des Tutors gehört es daher, solche Rückmeldekanäle zu öffnen bzw. zu konstitutieren.

Neben unterstützenden Faktoren für die Bildung neuer Konstrukte nennt Kelly auch hinderliche bzw. hemmende Bedingungen hierfür. Erwähnt sei hier die «ausschliessliche Beschäftigung mit altem Material» (S. 117), das sich als starre Gewohnheit bzw. zwanghaftes Verhalten äussern kann, und der Formierung neuer Vorstellung bzw. alternativer Interpretationen von Ereignissen im Wege steht.

Aus der «Theorie der persönlichen Konstrukte» heraus entwickelte Kelly (1955) ein umfangreiches Instrumentarium psychotherapeutischer Methoden. Bekannt wurde sein «Repetory Grid Test» als diagnostisches Verfahren und die «Fixed-Role-Therapy» als Interventionsmethode. Darüber hinaus beinhaltet der weniger verbreitete zweite Teil seines Monumentalwerkes «The Psychology of Personal Constructs» eine detaillierte Beschreibung eines umfassenden psychotherapeutischen Ansatzes. Da der Kern dieses Ansatzes in der Veränderung von Konstrukten und Konstruktsystemen besteht, kann er im Wesentlichen auch (wenn man anstelle einer Defizit- eine Wachstumsperspektive einnimmt) als ein didaktisches Arsenal für die Initiierung von Kompetenzentwicklungsprozessen interpretiert werden.

Eine Vielzahl der von Kelly vorgestellten Verfahren beziehen sich auf zwei Prozesse, die er als das Lockern («loosening») bzw. Festzurren («Tightening») von Konstruktionen beschreibt. Jeder kreative Prozess besteht aus einer Abfolge dieser beiden gegensätzlichen Abschnitte einer Konstruktsystemveränderung. Kelly verdeutlicht die Folgen der Unterschiedlichkeit dieser beiden kognitiven Prozesse: «Yet the creative circle is never complete until the idea has taken definite shape and the sturdiness of its structure tested in the rushing torrent of real events. The tragedy of so many of us is that we can perform only half the cycle - sometimes one half, sometimes only the other. What one man can imagine he cannot test, while another goes through life attempting only what others have imagined» (S. 1031).

\section{Methoden provokativer Didaktik in internetgestützten Lehr-Lern-}

\section{arrangements}

Provokative Statements in Online-Dikussionen

Die Erfahrungen zeigen, dass es für die Lehrenden gar nicht leicht ist, eine Online-Diskussion, die sich fachlich auf hohem Niveau bewegt und sich reger Beteiligung erfreut, in Gang zu setzen. Die Konfrontation der Lernenden mit interessanten Lernmaterialien führt in der Regel ebenso wenig zu einer solchen Diskussion wie die Verwendung allgemein und neutral gehaltener Fragestellungen zur Einleitung.

Baltes (2001) berichtet von den Problemen eines Online-Instruktors, dem es nicht gelingt, seine Student/innen zu einem aktiven Gedankenaustausch in Diskussionsforen zu bewegen. Sie gibt ihm den Rat, «aktivierende Fragen» (S. 98) zu verwenden. Ausserdem empfiehlt sie: «Spielen Sie den <advocatus diaboli〉: Fordern Sie von den Teilnehmern, dass diese ihre Argumente gegenüber verschiedenen Standpunkten rechtfertigen» (S.102). In ähnlicher Weise aktivierend auf die Lernenden wirkt die Verwendung provokativer Statements. Setzt man diese aber als Auftakt einer OnlineDiskussion mit einer grösseren Gruppe von Lernenden ein, so ist unbedingt darauf zu achten, dass die Provokation nicht nur kleine Teile der Gruppe anspricht. Gelungene provokative Statements

- schildern z. B. Sachverhalte aus einer Perspektive, die konträr ist zu der in der Gruppe einhellig vertretenen und regen damit zu Widerspruch an, oder

- sind bewusst ambivalent formuliert und fördern damit unterschiedliche Meinungen in der Gruppe zu Tage, die dann diskutiert werden können, oder

- übertreiben in der Gruppe vorherrschende Auffassungen (z. B. hinsichtlich einer Theorie) so masslos (z. B. durch Schilderung der Konsequenzen der alleinigen Anwendung dieser Theorie), dass von den Vertretern dieser Auffassung selbst Einschränkungen zur Gültigkeit und Reichweite vorgenommen werden (vgl. Beschreibungen provokativer Techniken bei Farrelly/ Brandsma 1974).

Darüber hinaus sind provokative Statements auch geeignet, um gezielt auf einzelne Beiträge in Online-Diskussionen zu antworten, wenn diese sehr einseitige oder eingeschränkte Sichtweisen oder Einstellungen widerspiegeln. Dabei ist darauf zu achten, dass die Provokation, da sie ja öffentlich stattfindet, nicht als persönlich beleidigend oder kränkend missinter- 
pretiert werden kann. Besonders geeignet ist hier wiederum die Technik der Übertreibung, evtl. gepaart mit einer guten Prise Humor.

\section{Die Auswahl einstellungskonträren Materials}

Der Lehrende kann den/die Lernenden nur dann mit einstellungskonträrem Material konfrontieren, wenn er über deren Ansichten, Einstellungen und Werte hinreichend informiert ist. Der erste Schritt einer provokativen Diagnostik ist daher die Diagnose der Einstellungen des Lernenden. Hierzu steht dem Lehrenden gerade im Bereich des internetgestützten Lernens eine Reihe von Techniken zur Verfügung:

- Online-Befragung (geschlossenes Antwort-Format)

In vielen Lernplattformen bzw. Diskussionsforen-Programmen lässt sich eine einfache Multiple-Choice-Befragung der Lernenden mit wenig Aufwand zusammenstellen. Oftmals ist allerdings lediglich eine anonyme Auswertung vorgesehen, so dass eine Individualdiagnostik nicht möglich ist. Bei nicht-anonymisierten Befragungen wächst andererseits die Gefahr der absichtlichen Ergebnisverfälschung bzw. der Teilnahmeverweigerung, insbesondere dann, wenn es sich persönliche bzw. emotional stark besetzte Einstellungen handelt.

- Online-Befragung (offene Fragen)

Der Online-Instruktor bzw. Tutor hat die Möglichkeit sich mit offenen Fragen entweder an eine ganze Lerngruppe oder an einen einzelnen Teilnehmer zu wenden. Offene Fragen sind weniger leicht verfälschbar, da eine Begründung der gegebenen Antwort verlangt werden kann. Die schriftliche Beantwortung offener Fragen beim online-basierten Lernen bietet dem Lehrenden gegenüber den verbalen Antworten beim Präsenzlernen bessere Analysemöglichkeiten. Darüber hinaus können die Äusserungen der Teilnehmer auch sehr viel später noch eingesehen werden.

- Analyse von Hintergrundinformationen

Oftmals lassen sich relevante Einstellungen und Ansichten eines Lernenden auch aus dessen Biographie bzw. gegenwärtigen Lebensumständen erahnen. Die Individualisierung des Lernens und der Betreuung durch den Lehrenden, verlangt, dass dieser sich auch mit solchen Informationen auseinandersetzt, insofern der Lernende bereit ist, diese preiszugeben.

- Analyse der Foren-/ Chatbeiträge

Die Einstellungen eines Lernenden lassen sich in den meisten Fällen auch ohne eine direkte, zielgerichtete Abfrage aus dessen Ausserungen im Rahmen entsprechender themen- bzw. problembezogener Diskussionen erschliessen. Onlinebasierte Forendiskussionen bieten dem Lehrenden oft die Möglichkeit, sämtliche Beiträge eines bestimmten Teilnehmers chronologisch zusammenzustellen und einzusehen. Bei sehr umfangreichem Material ist es meist möglich innerhalb der Beiträge nach bestimmten Stichworten zu suchen. Die Analyse von Foren- und Chatbeiträgen ist ein nonreaktives Verfahren, da der Teilnehmer zum Zeitpunkt der Abfassung des Beitrags nicht vorhersehen kann, dass der Lehrende dieses zu einem späteren Zeitpunkt verwendet, um seine Einstellungen bzw. Werte kennen zu lernen. Die diagnostischen Daten dieser Methode sind daher in besonderer Weise verfälschungsresistent und somit valide.

- Gruppenzusammenstellung

Die Zusammenstellung von Gruppen mit Teilnehmern unterschiedlicher Erfahrungshintergründe und Einstellungen kann einerseits unmittelbar als eine Methode sozialen Lernens eingesetzt werden, andererseits aber auch Diskussionen und Konflikte erzeugen, aus denen der Lehrende wertvolle Hinweise auf die Einstellungen und Werte der Gruppenangehörigen gewinnen kann

- Wenig bevorzugte Alternativen

Im Zusammenhang mit selbstgesteuertem Lernen erhält der Lernende häufig eine weitreichende Wahlfreiheit bezüglich der von ihm zu bearbeitenden Aufgaben bzw. Lernmaterialien. Stellt der Lehrende fest, dass diese Themenauswahl stets um eine bestimmte Theorie oder ein bestimmtes Paradigma kreist, so kann er daraus Rückschlüsse auf die Vorlieben bzw. Einstellungen des Lernenden ziehen.

Einschränkend ist zu bemerken, dass trotz der gerade im Bereich des ELearning verbesserten Diagnosemöglichkeiten, die Vermutung über bestimmte Einstellungen eines Lerners stets mit Unsicherheiten behaftet sein wird. Es dürfte in der Realität nie mit absoluter Sicherheit möglich sein, die Gefühle, Ansichten und Einstellungen eines anderen Menschen zu durchschauen. Eine weitere Fehlerquelle für die Auswahl einstellungskonträren Materials ergibt sich aus der Unmöglichkeit, die Widersprüchlichkeit zweier Theorien oder Anschauungen objektiv zu bemessen Wenn Theorie X für den Tutor subjektiv als konträr zu Theorie Y begriffen wird, ergibt sich daraus keineswegs, dass diese Einschätzung auch vom 
Lernenden geteilt wird. Nichtsdestotrotz wird durch die intendierte Auswahl einstellungskonträren Materials zweifellos die Wahrscheinlichkeit erhöht, kognitive Konflikte beim Lerner auszulösen, die letztlich zu einer Einstellungsänderung bzw. zu einem Wertewandel führen und somit Teil einer Kompetenzentwicklung sein können.

\section{Das Induzieren einstellungskonträrer Perspektiven}

In einem klassischen sozialpsychologischen Experiment von Cohen (1962) erhielten Studierende den Auftrag, in einem Aufsatz eine Position zu vertreten, die ihrer wirklichen Einstellung diametral entgegenstand. Während die Versuchspersonen in Wirklichkeit eine kritische Haltung zur örtlichen Polizei hatten, sollten sie innerhalb ihres Aufsatzes die positiven Aspekte der Polizeiarbeit darstellen und deren personelle Verstärkung fordern. Nach Abfassung des Aufsatzes wurde die tatsächliche Einstellung der Studierenden zur Polizei erfasst. Dabei stellte sich heraus, dass Studierende, die an dem Experiment teilgenommen hatten, nunmehr eine deutlich veränderte Einstellung äusserten: Im Vergleich zu Studierenden, die keinen Aufsatz geschrieben hatten, fiel ihre Bewertung der örtlichen Polizei nun deutlich positiver aus.

Entscheidend für das Ergebnis von Cohen war eine gewisse subjektive Freiwilligkeit der Probanden. Nahm man den Studierenden diese subjektive Wahlfreiheit der Teilnahme an dem Experiment, indem man ihnen z. B eine sehr hohe Belohnung versprach, so veränderte sich deren Einstellung zur Polizei nicht. Dieses experimentelle Setting, das als «forced complience»-Paradima bekannt ist, wurde vielfach variiert wiederholt. Es liess sich ganz allgemein feststellen, dass ein zunächst einstellungskonträres Verhalten, das subjektiv freiwillig ausgeführt wird, zu einer Angleichung der Einstellung hin zur Übereinstimmung mit diesem Verhalten führt.

Die Sozialpsychologie liefert unterschiedliche Theorien, die in der Lage sind, diese Einstellungsänderung zu erklären. Festinger (1957) beschreibt die Diskrepanz zwischen induzierter Meinungsäusserung und bisheriger Einstellung als kognitive Dissonanz. Diese wird vom handelnden Subjekt als unangenehmer Zustand wahrgenommen. Eine Möglichkeit zur Reduktion der Dissonanz besteht in der Anpassung der Einstellung an die im Aufsatz geäusserten Ansichten.

Eine alternative Erklärung für dieses Experiment liefert die Theorie der Selbstwahrnehmung nach Bem (1967). Diese Theorie stellte die weitverbreitete Ansicht, dass Einstellungen die Grundlage für Verhalten bilden in Frage, indem eine umgekehrte Kausalität postuliert wird: Verhalten verursacht Einstellungen. Nach Bem (1967) erschliessen Personen ihre eigenen Einstellungen in ähnlicher Weise, wie sie die Einstellungen anderer erschliessen: nämlich durch Beobachtung ihres Verhaltens. Die Versuchspersonen in Cohens Experiment hätten ihre neue Einstellung gegenüber der Polizei somit aus ihrem offenen Verhalten, d. h. aus den im Aufsatz geäusserten Aussagen geschlossen.

Eine alternative Interpretation der Ergebnisse Cohens kann aus der Perspektive der Theorie der persönlichen Konstrukte unternommen werden: Das experimentelle Setting zwang die Versuchspersonen, mit einem neuen Konstruktsystem gedanklich zu experimentieren. Selbstverständlich war den Probanden klar, dass die zu vertretenden Einstellungen sich zu ihrem eigenen, bisherigen Konstruktsystem mehr oder minder inkompatibel verhielten, dennoch bewirkte die Anweisung, sich so zu verhalten, als ob sie aus dem neuen Konstruktsystem heraus agierten, dass sie sich inhaltlich mit dem neuen System auseinander setzten.

Weiterhin verlangte die Instruktion, nicht nur ein gedankliches Durchdringen der neuen Konstrukte, sondern darüber hinaus, ein aktives Handeln aus diesen neuen Vorstellungen heraus. Um sich in der Experimentalsituation angemessen zu verhalten, erbrachte ein Rückgriff auf das bisherige Konstruktsystem kein erfolgsversprechendes Ergebnis, anstelle dessen war eine Handlungsplanung innerhalb dieses Settings nur durch Anwendung der neuen Vorstellungen möglich. Das Erfüllen der Experimentalaufgabe stellte somit eine erfolgreiche Anwendung der neu erworbenen Konstrukte dar. Es vollzog sich ein Prozess, der in ähnlicher Form auch ausserhalb experimentaler Settings die Grundlage für Einstellungsänderungen bildet: Das Scheitern bisheriger Konstrukte und die erfolgreiche Anwendung neuer Vorstellungen bei der Bewältigung einer realen Anforderung.

Der von Cohen experimentell erprobte Mechanismus lässt sich als Methode der provokativen Didaktik auch in einem Lehr-Lernarrangement als Mittel zur Veränderung von Einstellungen und Werten verwenden.

- Der Lehrende (Tutor) gibt eine neue Ansicht vor, die mit der bisherigen Ansicht des Lerners inhaltlich inkompatibel ist.

- Der Lerner soll dabei

- Material der Vertreter und Kritiker der neuen Ansicht zur Verfügung haben. 
- sich selbständig über einen längeren Zeitraum hinweg mit dem Material auseinandersetzen.

- die neue Ansicht schriftlich darlegen und vertreten.

- Argumente für die neue Ansicht finden.

- darstellen, welche Konsequenzen die neue Ansicht im Hinblick auf bereits bestehende Erfahrungen besitzt.

- Alltagserfahrungen mit Hilfe der neuen Ansicht erklären.

Advocatus diaboli: Das Vertreten einstellungskonträren Ansichten Der Lehrende stellt dem Lernenden eine Aufgabe, die intendiert erzwingt, dass dieser im Rahmen einer synchronen oder asynchronen Online-Diskussion Argumente findet und vorbringt, die seiner bisherigen Anschauung konträr entgegenstehen. Die konkrete Ausgestaltung der Aufgabe kann auf unterschiedliche Art und Weise erfolgen:

- Ein Anhänger einer Theorie X soll Argumente, Fallbeispiele oder empirische Forschungsergebnisse sammeln und innerhalb der Diskussion vorstellen, die eben diese Theorie widerlegen oder ihre Bedeutung in Frage stellen.

- Ein Gegner einer Theorie Y soll Argumente, Alltagsbeispiele oder Forschungsergebnisse zusammentragen und in der Diskussion vertreten, die die Bedeutung dieser Theorie unterstützten und die Nützlichkeit ihrer Anwendung unterstreichen.

- Anhänger der konträren Theorien X und Y sollen in einer forengestützten Diskussion jeweils die ihrer tatsächlichen Anschauung entgegengesetzte Theorie vertreten.

- Ein vorgegebenes, reales Fallbeispiel soll von Gegnern der Theorie X in den Begriffen eben dieser Theorie den anderen Teilnehmern der Diskussion erklärt werden.

Die Aufgaben bedürfen detaillierter Instruktionen. Bei der Aufgabendurchführung ist darauf zu achten, dass die Lernenden, wenn Sie die Aufgabe haben, eine an und für sich meinungskonträre Perspektive zu vertreten, nach Möglichkeit Argumente für diese Perspektive finden, die sie tatsächlich unterstützen. Verhindert werden sollte, dass lediglich oberflächlich Aussagen getroffen werden, von denen man annimmt, dass eben solche von Vertretern der entsprechenden Theorie geäussert werden. Die zentrale Aufgabe des Tutors bei den beschriebenen Aufgaben besteht daher darin, stets die «Echtheit» der vertretenen Argumentation einzufordern, indem von den
Lernenden geäusserte Argumente hinterfragt werden bzw. um deren Präzisierung gebeten wird.

Die Kommunikation zwischen Tutor und Lernendem folgt bei den beschriebenen Aufgaben somit einem komplizierten Muster: Einerseits weiss der Tutor ja, dass der Lernende nur höchst unwillig eben jene Ansichten vertritt, die er durch die Aufgabenstellung zu vertreten hat, andererseits ist er aber gezwungen, eben jene Ansichten herauszufordern, um die notwendige Realitätsnähe der Anforderungssituation zu erreichen.

Um die eigentliche Ansicht des Lernenden herauszufordern bzw. zu labilisieren, verlangt der Tutor von diesem, dass er eine meinungskonträre Perspektive einnimmt. Aber eben diese Perspektive muss der Tutor dann abermals herausfordern, um deren Einnahme durch den Lernenden «echt» werden zu lassen. Der Tutor vertritt nun also Argumente, die der Lernende eigentlich selbst unterstützen möchte. Lässt er sich aber auf diese Argumente ein, muss der Tutor unverzüglich und unmissverständlich klarstellen, dass hierdurch die Aufgabenstellung unzureichend erfüllt wird.

\section{Fixed-Role-Methode}

Ein fiktives Beispiel:

Ein Vorarbeiter, in dessen Arbeitsgruppe es zu häufigen Konflikten gekommen ist, und der von seinen Mitarbeitern als 〈engstirnig〉 und 〈autoritär〉 bezeichnet wird, nimmt an einer Weiterbildung zu Führungstechniken teil. Der Online-Coach dieses Teilnehmers gibt ihm eine Rollenspielaufgabe: «Der Vorarbeiter Herr Müller ist bei seinen Arbeitern beliebt. Sie beschreiben ihn als einen fairen Vorgesetzten, der immer ein offenes Ohr hat, wenn jemand ein ernstes Anliegen hat, der aber auch bereit ist, sich durchzusetzen, wenn es darum geht, dass die Arbeit mit der notwendigen Sorgfalt erledigt wird. Herr Müller trifft sich jeden Tag eine Viertelstunde vor der Schicht mit allen Mitarbeitern auf einen Kaffee. Bei Entscheidungen, die alle Angehörige der Arbeitsgruppe betreffen, erkundigt er sich in dieser Runde nach der allgemeinen Meinung. Hier erklärt er auch die jeweils anstehenden Aufgaben und diskutiert, wer welche Arbeit übernehmen soll. Wann immer Herr Müller den Eindruck hat, dass ein Arbeiter seiner Gruppe, Ideen haben könnte, wie man ein bestimmtes Problem löst, wendet er sich während der Arbeitszeit direkt an diesen Mitarbeiter.

Für die Erstellung des Urlaubsplans bittet Herr Müller alle Mitarbeiter kurz aufzuschreiben, wann sie am liebsten ihren Jahresurlaub antreten würden. Die Auswertung der Urlaubswünsche macht er öffentlich vor der Gruppe. 
Wenn bestimmte Zeiträume sich so überschneiden, dass die Urlaubsgenehmigung nicht möglich ist, werden die entsprechenden Arbeiter gebeten, einen Ersatztermin zu wählen. Wenn sich ein Mitarbeiter mit einem persönlichen Problem an Herrn Müller wendet, muss er nicht befürchten, sich lächerlich zu machen oder nicht ernst genommen zu werden. Herr Müller weiss, dass jeder Mensch seine ganz eigenen Merkwürdigkeiten, Stärken und Schwächen hat und versucht daher, die individuellen Potenziale jedes Einzelnen weiterzuentwickeln. Das hat dazu geführt, dass die meisten seiner Mitarbeiter sehr offen zu Herrn Müller sind. Man spricht mit ihm nicht nur über dienstliche Angelegenheiten, sondern auch über vielerlei private und allgemeine Dinge.»

Der Teilnehmer soll nun während seiner realen Tätigkeit als Vorarbeiter in die Rolle von Herrn Müller schlüpfen. In möglichst vielen Situationen soll der Teilnehmer in den folgenden Tagen bzw. Wochen, ausprobieren, sich nicht so wie bisher zu verhalten, sondern so wie Herr Müller es tun würde. Die hier beschriebene Methode wurde ursprünglich von Kelly (1955) als psychotherapeutische Technik entwickelt. Die «Verschreibung einer Rolle» oder «Fixed-Role-Therapy» stellt gewissermassen die extremste Form einer Konfrontation mit einstellungskonträrem Material dar: Der Lernende wird selbst zum Handelnden anhand eines vom Lehrenden entworfenen Rollenmodells. Der Lehrende entwirft bewusst eine Rolle, deren Handeln Aspekte beinhaltet, die den bisherigen Einstellungen und Werten des Lernenden konträr entgegenstehen.

Kelly (1955) nennt sechs Regeln für die Entwicklung einer Rolle im Rahmen eines Fixed-Role-Settings, die sich in seiner praktischen Arbeit empirisch als nützlich erwiesen haben:

1. «Development of a major theme other than correction of minor faults»

Die Rolle soll nicht nur eine kleinere Veränderung von Einstellungen, Verhalten und Werten des Klienten mit sich bringen, sondern einen grundsätzlich neuen Rahmen für ihn bieten.

2. «The use of sharp contrast»

Die Rolle soll dem bisherigen Verhalten diametral entgegengesetzt konstruiert sein. Das Erkunden neuer Einstellungen und Verhaltensweisen gelingt leichter, wenn diese sehr weit ausserhalb des bisherigen Konstruktsystems liegen.

3. «Setting ongoing processes in motion rather than creating a new state» Ziel der Fixed-Role-Aufgabe sollte es nicht sein, den Lernenden von einem starren, undurchlässigen Konstruktsystem, das ihn an Verän- derungen hindert, in ein anderes zu stossen. Er soll vielmehr lernen, sich zu verändern. Neue, durchlässigere Konzeptualisierungen sollen die bisherigen Konstrukte ersetzen.

4. «Testable hypotheses for the client»

Die neuen Vorstellungen, die der Proband mit der Rolle übernimmt, können sich nur dann verfestigen, wenn sich ihre Anwendung in der Praxis als sinnvoll erweist. Das Konstruktsystem der neuen Rolle muss sich daher rasch in der Praxis bewähren können.

5. «Emphasis upon role perceptions»

Die Rolle soll nicht lediglich eine Aufzählung von Verhaltensweisen beinhalten. Sie soll ein Konzeptgerüst für ein neues Verständnis bilden.

6. «The protective mask»

Die Rolle soll dem Klienten einen geschützten Rahmen bieten, um neue Vorstellungen und Verhaltensweisen auszuprobieren.

Die verschriebene Rolle gibt dem Lernenden die Möglichkeit, ein neues Verhalten und das damit verbundene Konstruktsystem im realen Funktionsumfeld zu erproben. Er hat dadurch die Möglichkeit, Erfahrungen zu sammeln, die auf andere Art und Weise nicht zu erlangen wären. Die neuen Erfahrungen können das neue Konstruktsystem und damit neue Einstellungen und Werte stabilisieren und ausgestalten.

Emotionen des Lernenden überwachen

Einstellungsänderungsprozesse können von erheblichen Emotionen begleitet sein. Der Online-Tutor bzw. -Instruktor übernimmt durch die Evozierung dieser Emotionen (aufgrund der provokativen Intervention) eine besondere Verantwortung für den Lernenden. Im Einzelnen muss der Lehrenden mit folgenden Affekten des Lernenden rechnen:

- die aggressive Abwehr einstellungskonträren Materials oder entsprechender Äusserungen anderer Kursteilnehmer,

- Angst des Lerners vor Bedrohungen der eigenen Einstellungen,

- Scham bei der Aufdeckung von Fehlannahmen,

- Traurigkeit/Depression, wenn ein selbstwertstützendes Konstruktsystem zerstört wurde.

Das emotionale Erleben eines Lernenden kann durchaus zu einer Belastung für andere Kursteilnehmer bzw. den Lehrenden selbst werden. Es kann zu Feindseligkeiten gegenüber den Mitlernenden oder den Tutor kommen. Der Lerner kann sich beim Veranstaltungsträger beschweren oder er kann versuchen, seine emotionalen Probleme zu verbergen bzw. zu leugnen. 
Der Einsatz der provokativen Didaktik verlangt von der Lehrkraft sehr viel Fingerspitzengefühl. Das Verhältnis zwischen Lernendem und Lehrendem wird persönlicher und individueller als in herkömmlichen Lehr-Lernarrangements. Der Lehrende wird zu einem Coach oder gar «Therapeuten», der Verantwortung für den Entwicklungsprozess seines «Klienten» übernimmt, der bemüht ist, dessen Kompetenzentwicklungsprozess zu verfolgen, ihn zu unterstützen und immer wieder neue, provokative Herausforderungen und Anregungen für ihn zu konstruieren.

\section{Literatur}

Anderson, C./Roth, K. (1989): The search for school climate: A review of research. Review of Educational Research, 52, S. 368-420.

Anderson, C./Smith, E. (1987): Teaching science. In: V. RichardsonKoehler (Hrsg.): Educator's Handbook (S. 84-111). New York.

Arnold R./Lipsmeier A./Ott, B.(1998): Berufspädagogik kompakt. Berlin.

Balacheff, N. (1991): Treatment of refutations: Aspects of the complexity of a constructivist approach to mathematics learning. In: von Glasersfeld,

E. (Hrsg.): Radical constructivism in mathematics education. Dordrecht, S. 89-110.

Baltes, B. (2001): Online-Lernen. Schwangau.

Baxter, J. (1995): Children's understanding of astronomy and the earth sciences. In: Glynn, S. M./Duit, R. (Hrsg.): Learning Science in the Schools: Research Reforming Practice. Mahwah, NJ., S. 155-177.

Bell, A. (1983): Diagnostic teaching of additive and multplicative problems. In: Hershkowitz, R. (Hrsg.): Proceedings of the Seventh International Conference for the Psychology of Mathematics Education. Rehovot, Israel, S. 205-210.

Bem, D. J. (1967): Self-perception: An alternative interpretation of cognitive dissonance phenomena. Psychological Review, 74, S. 183-200.

Cohen, A. R. (1962): An experiment on small rewards for discrepant compliance and attitude change. In: Brehm, J. W./ Cohen, A. R.: Explorations in Cognitive Dissonance. Wiley, New York, 1962.

Dohmen, G./Knoll, J./Siebert, H. (2001): Selbstgesteuertes Lernen ohne Lehre? In: Heuer, U./Botzat, T./Meisel, K. (Hrsg.): Neue Lehr- und Lernkulturen in der Weiterbildung. Bielefeld, S. 158-183.

Draschoff, S. (2000): Lernen am Computer durch Konfliktinduzierung. Münster

Duggleby, J. (2000): How to be an Online Tutor. Burlington.
Duit, R. (1995): Zur Rolle der konstruktivistischen Sichtweise in der naturwissenschaftsdidaktischen Lehr- und Lernforschung. Zeitschrift für Pädagogik, 41, S. 905-923.

Erpenbeck, J./Heyse, V. (1999): Die Kompetenzbiographie: Strategien der Kompetenzentwicklung durch selbstorganisiertes Lernen und multimediale Kommunikation. Münster.

Farrelly, F./Brandsma, J. (1974): Provocative Therapy. Cupertino, CA.

Festinger, L. (1957): A Theory of Cognitive Dissonance. Stanford, CA.

Fishbein, M. (1967): Attitude and the prediction of behaviours. In: Fishbein, M. (Hrsg.): Readings in Attitude Theory and Measurement. New York.

Gehrlicher, I. (2001): Modularisierung. In: Hanft, A. (Hrsg.): Grundbegriffe des Hochschulmanagements. Neuwied, S. 291-294.

Giesecke, H. (1973): Methodik des politischen Unterrichts. München.

Haken, H. (1988): Erfolgsgeheimnisse der Natur. Synergetik: Die Lehre vom Zusammenwirken. Frankfurt.

Herkner, W. (1988): Einführung in die Sozialpsychologie. Bern.

Heuer, U. (2001): Lehren und Lernen im Wandel. In: Heuer, U./ Botzat, T./ Meisel, K. (Hrsg.): Neue Lehr- und Lernkulturen in der Weiterbildung. Bielefeld, S. 13-35.

Jarvilehto, T. (2000): Feeling as knowing: I. Emotion as reorganization of the organism-environment system. Consciousness \& Emotion 1(2), S. 57.

Johnston, K./Scott, P. (1991): Diagnostic teaching in the science classroom: Teaching/learning strategies to promote development in understanding about conservation of mass on dissolving. Research in Science \& Technological Eduacation, 9, S. 193-212.

Kelly, G. A. (1955): The Psychology of Personal Constructs: A Theory of Personality (Vol. 1 and 2). London.

Kunert, K./Zitterbart, E. (1978): Provokation im Unterricht. Donauwörth. Maturana, H. R./Varela, F. (1987). Der Baum der Erkenntnis: Die biologischen Wurzeln des menschlichen Erkennens. Bern.

Meyer, H. (2000). Unterrichtsmethoden. Frankfurt. Bd. 2. Praxisband.

Mills, J. (1996): A Provocative Pedagogy. Paper Presentation. 11th International Workshop Conference on Teaching Philosophy. Norfolk, VA, July, 31-Aug., S. 4.

Mills, J./Aronson, E./Robinson, H. (1959): Selectivity in exposure to information. Journal of Abnormal and Social Psychology, 59, S. 250-253. 
MedienPädagogik

Müskens, I. (2001): Selbstverständnis, Aufgaben und Funktion von Tutoren in Online- Lernumgebung. In AUE-Informationsdienst Hochschule und Weiterbildung, 2/01, S. 29-34.

Peters, W. (1911): Gefühl und Erinnerung. Psychologische Arbeiten, 6, S. 197-260.

Petty, R. E./ Cacioppo, J. T. (1981): Attitudes and Persuasion: Classic and Contemporary Approaches. Dubuque.

Rautenstrauch, C. (2001): Tele-Tutoren: Qualifizierungsmerkmale einer neu entstehenden Profession. Bielefeld.

Rosenberg, M. J./ Hovland, C. I. (1960): Cognitive, affective, and behavioral components of attitudes. In: Hovland, C. I./ Rosenberg, M. J.(Hrsg.): Attitude Organization and Change: An Analysis of Consistency among Attitude Components. New Haven, CT, S. 1-14.

Roth, K. J./ Anderson, C. W. (1988): Promoting conceptual change learning from science textbooks. In: Ramsden, P. (Hrsg.): Improving Learning: New Perspectives. London, S. 109-141.

Siebert, H. (1996): Didaktisches Handeln in der Erwachsenenbildung. Neuwied.

Siebert, H. (1999): Pädagogischer Konstruktivismus: Eine Bilanz der Konstruktivismusdebatte für die Bildungspraxis. Neuwied.

Staudt, E./ Kriegsmann, B. (1999): Weiterbildung: Ein Mythos zerbricht. In Kompetenzentwicklung '99: Aspekte einer neuen Lernkultur Argumente, Erfahrungen, Konsequenzen. Münster, S. 17-60.

Turner, R. H./ Barlow, J. H. (1951): Memory for pleasant and unpleasant experiences: Some methodological considerations. Journal of Experimental Psychology, 42, S. 189-196.

Underhill, R. G. (1991): Two layers of constructivist curricular interaction. In: von Glasersfeld, E. (Hrsg.): Radical constructivism in mathematics education. Dordrecht, S. 229-248.

Vosniadou, S. (1994): Capturing and modeling the process of conceptual change. Learning and Instruction, 4, S. 45-69.

Westera (2001): Competencies in education: a confusion of tongues. Journal of Curriculum Studies, 33, S. 5-88. 